\title{
Serum ferritin as a biomarker for NAFLD: ready for prime time?
}

\author{
Raj A. Shah ${ }^{1} \cdot$ Kris V. Kowdley ${ }^{1}$
}

Received: 1 November 2018 / Accepted: 22 January 2019 / Published online: 9 February 2019

(c) Asian Pacific Association for the Study of the Liver 2019

\begin{tabular}{|c|c|}
\hline \multicolumn{2}{|c|}{ Abbreviations } \\
\hline ALT & Alanine aminotransferase \\
\hline AST & Aspartate aminotransferase \\
\hline AUROC & $\begin{array}{l}\text { Area under the receiver operating character- } \\
\text { istic curve }\end{array}$ \\
\hline BMI & Body mass index \\
\hline FIB-4 & Fibrosis-4 score \\
\hline HSI & Hepatic steatosis index \\
\hline KNHANES & $\begin{array}{l}\text { Korean National Health and Nutritional } \\
\text { Examination Survey }\end{array}$ \\
\hline NAFLD & Non-alcoholic fatty liver disease \\
\hline NASH & Non-alcoholic steatohepatitis \\
\hline NHANES & $\begin{array}{l}\text { (US) National Health and Nutritional } \\
\text { Examination Survey }\end{array}$ \\
\hline NLFS & $\begin{array}{l}\text { Non-alcoholic fatty liver disease liver fat } \\
\text { score }\end{array}$ \\
\hline TIBC & Total iron-binding capacity \\
\hline ULN & Upper limit of normal \\
\hline
\end{tabular}

Non-alcoholic fatty liver disease (NAFLD), which is closely associated with type 2 diabetes and obesity [1,2], is now a major cause of liver disease in many countries and has contributed to an increase in liver disease-related mortality [3, 4]. According to the most recent practice guidance published by the American Association for the Study of Liver Diseases (AASLD) [5], the diagnosis of NAFLD is established by fulfillment of two criteria: evidence of hepatic steatosis by either imaging or histology and the exclusion of secondary etiologies such as significant alcohol ingestion, use of a medication known to cause steatosis, or liver disorders resulting in secondary steatosis such as hepatitis C, Wilson's disease, and inborn errors of metabolism. Furthermore, the differentiation of NAFLD into either non-alcoholic fatty liver (NAFL) or non-alcoholic steatohepatitis (NASH) can

Kris V. Kowdley

kris.kowdley@swedish.org

1 Liver Care Network and Organ Care Research, Swedish Medical Center, 1124 Columbia Street, Suite 600, Seattle, WA 98104, USA only be made on histologic analysis of liver tissue [5, 6]. This distinction is particularly significant as NASH is associated with an increased risk of cirrhosis, hepatocellular carcinoma, and liver-related mortality [1, 2, 4, 7]. The use of liver biopsy is restricted by cost, risk of complications, and patient discomfort [8], while diagnostic yield is limited by sampling variability and physician expertise [9]. Therefore, there is a need for non-invasive means for diagnosis of NASH and advanced fibrosis among patients with NAFLD. Imaging modalities such as ultrasonography, computed tomography, magnetic resonance imaging, and magnetic resonance spectroscopy have been reviewed by Lee et al. [10]. In addition, a number of blood-based tests have been examined for diagnosis and staging of NAFLD. Two indices that have demonstrated accuracy for detection of steatosis are the NAFLD Liver Fat Score (NLFS) and Hepatic Steatosis Index (HSI), components of which include the diagnosis of metabolic syndrome, diabetes mellitus, fasting serum insulin, AST, and AST/ALT ratio [11, 12]. The NLFS has a sensitivity of $86 \%$ and specificity of $71 \%$ at a threshold of -0.640 [11]. The HSI has a sensitivity of $66 \%$ and specificity of $69 \%$ at a cutoff of 36 [12].

Liver fibrosis stage is the strongest predictor for diseasespecific mortality in NAFLD [13]. Other than liver biopsy, radiologic modalities such as ultrasonography and transient elastography can be used to assess for fibrosis [14]. The Fibrosis-4 (FIB-4) score has also been developed as a calculation to assess for liver fibrosis using age, platelet count, ALT, and AST [7]. A score threshold of 2.67 has shown a sensitivity of $27 \%$ and specificity of $97 \%$ for fibrosis in NAFLD [14].

Ferritin expression is influenced by body's iron stores, but also by inflammation, and elevated ferritin levels are common in NAFLD [15]. In a study of 628 patients enrolled in the NASH Clinical Research Network (CRN) [16], we previously showed that a serum ferritin level $>1.5$ times the upper limit of normal (ULN) was independently associated with an increased risk of advanced fibrosis. Moreover, serum ferritin $>1.5 \times$ ULN was also found to be associated with increased likelihood of NASH diagnosis, higher steatosis grade, lobular inflammation, and hepatocellular ballooning. 
NAFLD, and especially NASH, is associated with increased levels of serum markers of oxidative damage and necrosis such as malondialdehyde and cytokeratin 18 (CK18) as well as decreased levels of the antioxidant thioredoxin-1 [17]. Kupffer cells play a role in liver inflammation and fibrosis in NAFLD via the release of cytokines and proteolytic enzymes, the recruitment of neutrophils and natural killer cells, and the endocytosis of cell debris [18]. Soluble CD163 (sCD163), a serum marker of Kupffer cell activation, has been associated with severity of fibrosis in NAFLD and independently associated with CK18, indicating that apoptosis may contribute to macrophage activation [19]. While this inflammatory cascade has not been linked to iron overload in NAFLD, markers of iron overload are associated with sCD163 in severe alcoholic hepatitis [20].

In this issue of Hepatology International, Jung et al. [21] examine the utility of serum ferritin to identify NAFLD using data from 25,597 participants in the Korean National Health and Nutritional Examination Survey (KNHANES). The population was divided by sex and then divided further into quartiles by ferritin levels. The participants in each subset with NLFS $\geq-0.640$ were considered likely to have NAFLD. A separate analysis of the population was performed whereby those with HSI $\geq 36$ were deemed likely to have NAFLD. Imaging or liver biopsy was not obtained as a part of KNHANES. The study found that participants in the highest quartile of serum ferritin levels were more likely to have NAFLD, based on NLFS or HSI. The odds ratio varied from 2.51 to 2.94 based on the index used and on sex (Fig. 1). Moreover, a rise in serum ferritin of $10 \mathrm{ng} / \mathrm{mL}$ increased the likelihood of NAFLD by 3-10\%, varying again with sex and index used. The association of ferritin levels was also examined in relation to ALT elevation, defined as $>40 \mathrm{IU} / \mathrm{L}$ for males and $>31 \mathrm{IU} / \mathrm{L}$ for females. A $10 \mathrm{ng} /$ $\mathrm{mL}$ increase in ferritin was associated with an increased likelihood of ALT elevation by $7 \%$ in males and $13 \%$ in females.
The odds of ALT elevation in the highest ferritin quartile were 4.08 for males and 5.72 for females. The study also examined the relationship between serum ferritin levels and FIB-4 score as a surrogate for liver fibrosis. An odds ratio of 9.67 for FIB-4 elevation was found in the highest ferritin quartile in females and 2.47 among males.

Metabolic syndrome and diabetes mellitus have previously been shown to associate with serum ferritin elevation [22]. The highest ferritin quartile was similarly associated with increased BMI, waist circumference, fasting glucose, and insulin levels in the current study, along with total cholesterol and triglycerides levels, while HDL cholesterol levels were lower. Alcohol intake was associated with ferritin levels, but the difference was only $2.6 \mathrm{~g} /$ day between the highest and lowest quartiles, amounting to less than half of a drink. Higher ferritin quartiles were associated with increased iron and transferrin saturation, and decreased total iron-binding capacity (TIBC).

Limitations of the study by Jung et al. should be noted. Imaging or invasive biopsy was not used to confirm the presence of NAFLD. The FIB-4 threshold of 2.67 for liver fibrosis has been examined in NAFLD [14], but FIB-4 in a general population may range from 0.90 to 4.5 [23]. While alcohol use was surveyed by KNHANES, medication history and pre-existing liver disease could not be included in the analysis. Furthermore, the authors did not examine the utility of isolated ferritin elevation to predict NAFLD as determined by NLFS or HSI.

This important study of a national population cohort confirms that elevated serum ferritin is associated with noninvasive markers for NAFLD. Moreover, it demonstrates an association between the degree of ferritin elevation and likelihood of ALT elevation. The analysis also suggests that higher ferritin levels may indicate hepatic fibrosis. The strength of these findings lies in the size of the cohort, which is 25,597 participants. Given these results, in the clinical

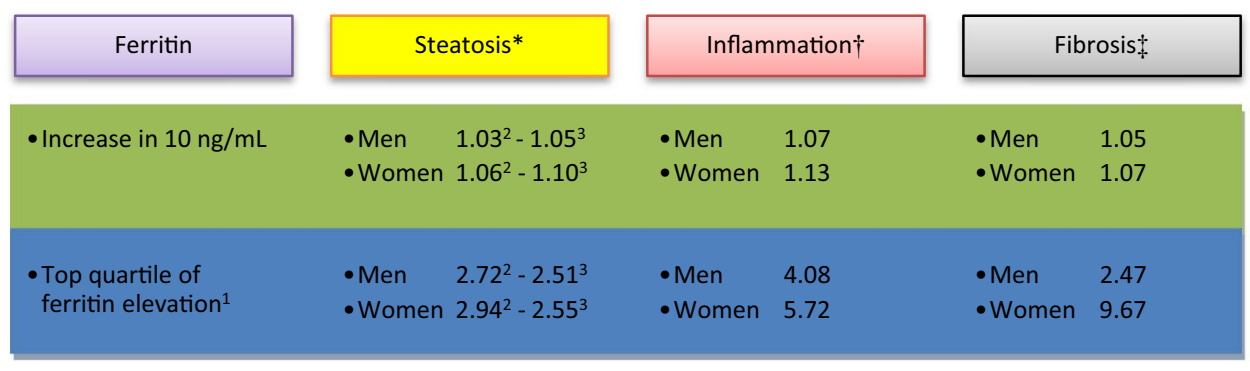

Fig. 1 Highlighted associations found in the study by Jung et al. The odds ratios for markers of hepatic steatosis, inflammation, and fibrosis separated by sex are listed. * Multivariable-adjusted odds ratios for hepatic steatosis, as defined by HSI or NLFS. † Multivariableadjusted odds ratios for hepatic inflammation, as demonstrated by ALT elevation. $\ddagger$ Multivariable-adjusted odds ratios for liver fibrosis, as defined by FIB-4 $\geq 2.67$. ${ }^{1}$ Top quartiles in this study are the following ranges of ferritin levels. In males: $147.12-966.4 \mathrm{ng} / \mathrm{mL}$, in females: $64.51-969.06 \mathrm{ng} / \mathrm{mL}$. ${ }^{2}$ For calculation of these odds ratios, hepatic steatosis was defined by HSI $\geq 36$. ${ }^{3}$ For calculation of these odds ratios, hepatic steatosis was defined by NLFS $\geq-0.640$ 
setting, measurement of ferritin may be considered to assess for risk of NAFLD in otherwise equivocal cases.

Additional long-term studies are warranted to evaluate the relationship between serum ferritin levels and complications of liver disease such as hepatocellular carcinoma and liverrelated mortality. Further elucidation of the pathophysiology of iron overload and inflammation in NAFLD may also identify targets for therapy.

\section{References}

1. Vernon G, Baranova A, Younossi ZM. Systematic review: the epidemiology and natural history of non-alcoholic fatty liver disease and non-alcoholic steatohepatitis in adults. Aliment Pharmacol Ther 2011;34(3):274-285

2. Musso G, Gambino R, Cassader M, Pagano G. Meta-analysis: natural history of non-alcoholic fatty liver disease (NAFLD) and diagnostic accuracy of non-invasive tests for liver disease severity. Ann Med 2011;43(8):617-649

3. Younossi Z, Tacke F, Arrese M, et al. Global perspectives on non-alcoholic fatty liver disease and non-alcoholic steatohepatitis. Hepatology 2018. https://doi.org/10.1002/hep.30251

4. Stepanova M, Rafiq N, Makhlouf H, et al. Predictors of all-cause mortality and liver-related mortality in patients with non-alcoholic fatty liver disease (NAFLD). Dig Dis Sci 2013;58(10):3017-3023

5. Chalasani N, Younossi Z, Lavine JE, et al. The diagnosis and management of nonalcoholic fatty liver disease: practice guidance from the American Association for the Study of Liver Diseases. Hepatology 2018;67(1):328-357

6. Marchesini G, Day CP, Dufour JF, et al. EASL-EASD-EASO clinical practice guidelines for the management of non-alcoholic fatty liver disease. J Hepatol 2016;64(6):1388-1402

7. Stål P. Liver fibrosis in non-alcoholic fatty liver disease-diagnostic challenge with prognostic significance. World J Gastroenterol 2015;21(39):11077-11087

8. Procopet B, Bureau C, Métivier S, et al. Tolerance of liver biopsy in a tertiary care center: comparison of the percutaneous and the transvenous route in 143 prospectively followed patients. Eur J Gastroenterol Hepatol 2012;24(10):1209-1213

9. Rockey DC, Caldwell SH, Goodman ZD, Nelson RC, Smith AD. Liver biopsy. Hepatology 2009;49(3):1017-1044

10. Lee SS, Park SH. Radiologic evaluation of nonalcoholic fatty liver disease. World J Gastroenterol 2014;20(23):7392-7402
11. Kotronen A, Peltonen M, Hakkarainen A, et al. Prediction of non-alcoholic fatty liver disease and liver fat using metabolic and genetic factors. Gastroenterology 2009;137(3):865-872

12. Cheung CL, Lam KS, Wong IC, Cheung BM. Non-invasive score identifies ultrasonography diagnosed non-alcoholic fatty liver disease and predicts mortality in the USA. BMC Med 2014;12(1):1-11

13. Ekstedt M, Hagström H, Nasr P, et al. Fibrosis stage is the strongest predictor for disease-specific mortality in NAFLD after up to 33 years of follow-up. Hepatology 2015;61(5):1547-1554

14. Xiao G, Zhu S, Xiao X, Yan L, Yang J, Wu G. Comparison of laboratory tests, ultrasound, or magnetic resonance elastography to detect fibrosis in patients with nonalcoholic fatty liver disease: a meta-analysis. Hepatology 2017;66(5):1486-1501

15. Dever JB, Mallory MA, Mallory JE, Wallace D, Kowdley KV. Phenotypic characteristics and diagnoses of patients referred to an iron overload clinic. Dig Dis Sci 2010;55(3):803-807

16. Kowdley KV, Belt P, Wilson LA, et al. Serum ferritin is an independent predictor of histologic severity and advanced fibrosis in patients with nonalcoholic fatty liver disease. Hepatology 2012;55(1):77-85

17. Maliken BD, Nelson JE, Klintworth HM, Beauchamp M, Yeh MM, Kowdley KV. Hepatic reticuloendothelial system cell iron deposition is associated with increased apoptosis in nonalcoholic fatty liver disease. Hepatology 2013;57(5):1806-1813

18. Baffy G. Kupffer cells in non-alcoholic fatty liver disease: the emerging view. J Hepatol 2009;51(1):212-223

19. Kazankov K, Barrera F, Møller HJ, et al. The macrophage activation marker $\mathrm{sCD} 163$ is associated with morphological disease stages in patients with non-alcoholic fatty liver disease. Liver Int 2016;36(10):1549-1557

20. Maras JS, Das S, Sharma S, et al. Iron-overload triggers ADAM17 mediated inflammation in severe alcoholic hepatitis. Sci Rep 2018;8(1):10264

21. Jung JY, Shim J-J, Park SK, et al. Serum ferritin level is associated with liver steatosis and fibrosis in Korean general population. Hepatol Int 2018. https://doi.org/10.1007/s12072-018-9892-8

22. Ford ES, Cogswell ME. Diabetes and serum ferritin concentration among US adults. Diabetes Care 1999;22(12):1978-1983

23. Zhang GM, Xia YJ. Reference intervals of fibrosis index based on four indicators in healthy elderly Chinese. J Clin Lab Anal 2016;30(5):645-648

Publisher's Note Springer Nature remains neutral with regard to jurisdictional claims in published maps and institutional affiliations. 